\title{
Correspondence
}

\section{Impact of improved perinatal care on the causes of death}

Sir,

We would like to reply to the correspondence provoked by our paper on the 'Impact of improved perinatal care on the causes of death'.'

We are happy to reassure Davis et $a^{2}$ that we are not confused by our own data. Such confusion as may exist seems to be more in the concept that changes in neonatology can, in large measure, be accounted for by changes in obstetrics. The increase in numbers of low birthweight babies reflects the increased readiness of obstetricians to intervene in pregnancies seen to be in jeopardy. We could not relate this to any known change in the hospital population, nor do we think that this change in obstetric policy is peculiar to our hospital. Few obstetricians would wish to explain the increased incidence of caesarean section in the United Kingdom generally on the basis of an increased incidence of obstetric disorders. The omission of data of specific lethal disease in the infants was deliberate. The attraction of Wiggleworth's categorisation on deaths is its clinical implications. Supplying all the data on the multiple pathologies found in most neonatal deaths tends to fascinate pathologists but genuinely confuse those clinicians preoccupied by treatment policies.

We welcome the additional data supplied by Sims and Chiswick relating to our own hospital but note that in infants weighing 1 to $1.5 \mathrm{~kg}$ since 1977 there has been a remarkable increase in survivors who did not require ventilation (Fig. 2) ${ }^{3}$ It seems likely that this is one more consequence of improved obstetric care. Indeed one wonders how many of those infants who survived only after ventilation would in fact have succumbed if birth asphyxia had been greater.

\section{References}

1 Barson AJ, Tasker M, Lieberman BA, Hillier VF. Impact of improved perinatal care on the causes of death. Arch Dis Child 1984;59:199-207.

2 Davis JA, Gandy GM, Morley CJ, Roberton NRC. Impact of improved perinatal care on the causes of death (letter). Arch Dis Child 1984;59:689.

${ }^{3}$ Sims DG, Chiswick ML. Impact of improved perinatal care on the causes of death (letter). Arch Dis Child 1984;59:689-90.

A J Barson, B A Lieberman, and M TASker St Mary's Hospital, Manchester M13 OJH

\section{Toe walking and neuromuscular disease}

Sir,

Illingworth and Illingworth are to be congratulated on their excellent article entitled 'Mothers are easily worried'. ${ }^{1}$ I would, however, like to comment on one point. Although toe walking is frequently a transient occurrence in a normal child, it can be the first sign of a serious neuromuscular disease that may not be easily detected, as Illingworth and Illingworth state, by 'simple clinical examination'. Simple clinical examination by those to whom children usually first present repeatedly fails to detect the early signs of serious neuromuscular disease such as Duchenne muscular dystrophy. ${ }^{2}$

Over a three year period, 18 patients with so called idiopathic toe walking were seen in this department, excluding patients with central nervous system disturbance, emotional disorders, and otherwise normal children with no tightening of the Achilles tendon. Over the same period there were 18 children whose toe walking had been the only or the major early symptom of a serious neuromuscular disease (muscular dystrophies (six); peroneal muscular atrophy (five); other neuropathies (four); unclassified (three)). Two patients (one with Becker dystrophy, one with peroneal muscular atrophy) were previously thought by paediatric specialists to be 'idiopathic' toe walkers. Looked at from another perspective, a recent study in this department showed that eight of 16 patients with Becker dystrophy and 13 of 44 patients with peroneal muscular atrophy presented either entirely or predominantly because of toe walking.

The correct diagnosis in many patients with neuromuscular disease is delayed because of a failure of practitioners to recognise the importance of the early gait abnormalities of these conditions. The average diagnostic delay, timed from the first documented medical contact with a symptom or sign that might have been recognised to be due to neuromuscular disease to the time of making the correct diagnosis, was 16 months (range up to five years) in 54 boys in Victoria, Australia with Duchenne muscular dystrophy (Shield LK, 1984, unpublished data). In Britain ${ }^{2}$ and the United States ${ }^{3}$ the time between initial parental concern and final diagnosis has been 2.5 years and 3.0 years respectively. Most parents want to know if there is something wrong with their child as soon as possible ${ }^{2}$ but the real tragedy in a delayed diagnosis is the potentially preventable birth of a second affected child with a disorder such as Duchenne muscular dystrophy.

Patients with early signs of disease usually present to practitioners who have the least experience in the diagnosis of neuromuscular diseases in childhood. Until general practitioners, paediatricians, and orthopaedic surgeons accept that toe walking may be the earliest sign of an underlying genetically determined neuromuscular disorder, diagnostic delay and family tragedies will continue to occur. With few exceptions, a serum creatine kinase determination should be performed on all toe walkers not easily explained by upper motor neurone disease or by emotional disturbance, etc. Further assessment is particularly indicated, even in the absence of overt weakness or deep tendon reflex abnormality, if there have been delayed milestones, the onset of toe walking after 3 years of age, frequent falling, or any other overt walking or running gait disturbance.

L K SHIELD Royal Children's Hospital, Parkville, Victoria 3052, Australia 